\title{
A Matroid on Hypergraphs, with Applications in Scene Analysis and Geometry
}

\author{
Walter Whiteley* \\ Champlain Regional College, 900 Riverside Drive, St. Lambert, Quebec, Canada J4P-3P2
}

\begin{abstract}
Following a conjecture of Sugihara, we characterize, combinatorially, the plane pictures of vertices and faces which lift to sharp three-dimensional scenes with plane faces. We also prove two generalizations of Laman's theorem on infinitesimally rigid plane frameworks. Both results are special cases of a representation theorem for the $k$-plane matroid of an incidence graph $G=(A, B ; I)$. The independent sets of incidences are characterized by $\left|I^{\prime}\right| \leq\left|A^{\prime}\right|+k\left|B^{\prime}\right|-k$ for all nonempty subsets, and the incidences are represented by rows of a matrix which uses indeterminate points in $k$-space for the vertices in $A$. Underlying this result is the simpler depth $k$ matroid of a hypergraph $H=(V, E)$ in which an independent set of edges satisfies $\left|E^{\prime}\right| \leq\left|V^{\prime}\right|-k$ for all nonempty subsets.
\end{abstract}

\section{Introduction}

A plane picture of a spatial polyhedral scene of plane faces and points of contact shows the projection of the designated points and the abstract incidence structure $S=(V, F ; I)$ of the vertices $V$ and the faces $F$. Given an incidence structure $S$ and a plane picture $P$ of the vertices, two key questions arise:

(a) Are there nontrivial (sharp) scenes projecting to the picture, with some (all) faces in distinct planes?

(b) If yes, what is the dimension of the vector space of scenes projecting to this picture?

For a specific picture, the answers depend on the geometry (or algebra) of the plane points, since the space of scenes is the solution space of a matrix

\footnotetext{
* Work supported, in part, by a grant from NSERC Canada. Preparation of the manuscript was supported, in part, by a grant from FCAR (Quebec).
} 
equation $A(S, P) \times Z=0$. If we fix the incidence structure, and vary the picture, certain minimum or generic answers occur for almost all pictures. These generic answers depend only on the combinatorics of the incidence structure.

Sugihara [9] conjectured a characterization of the incidence structures whose generic pictures lift to sharp scenes. Writing $i$ for $|I|, v$ for $|V|$, and $f$ for $|F|$, he conjectured:

A generic picture of an incidence structure lifts to a sharp polyhedral scene if and only if $i^{\prime} \leq v^{\prime}+3 f^{\prime}-4$ for all sets $I^{\prime}$ on at least two faces.

Sughihara proved this conjecture for special classes, such as 3-connected spherical polyhedra [9]. We verify the full conjecture, and the extension:

A generic picture of an incidence structure has a space of scenes (almost all sharp) of dimension $d$, with one face fixed, if and only if $i=v+3 f-3-d$, and $i^{\prime} \leq v^{\prime}+3 f^{\prime}-4$ for all sets $I^{\prime}$ on at least two faces.

Although these criteria appear to give exponential algorithms (involving a check of almost all subsets $I^{\prime}$ ), they actually yield polynomial algorithms of the order $O\left(i^{2}\right)[6],[10]$.

Our proof amounts to a controlled row reduction of the standard matrix for $A(S, P)$ to a block of rows on the variables for the heights of the vertices. Each row in the block has four entries, under four vertices sharing a face in the structure and this matrix defines the simpler depth $k$ matroid of the hypergraph $H=(V ; E)$. Independent sets of rows (or edges of the hypergraph) are characterized by the count

$$
e^{\prime} \leq v^{\prime}-3 \quad \text { for all nonempty subsets of edges }
$$

This characterization of the block is derived from a threefold truncation of the standard representation of the transversal matroid of a hypergraph, in which a set of edges is independent if and only if $e^{\prime} \leq v^{\prime}$ for all subsets of edges.

Our proof, presented in Sections 2 and 3, extends to $(k-1)$-space pictures of hyperplane and vertex scenes in $k$-space, for all positive $k$. In Section 4 we summarize this pattern, confirming our previous conjecture [17]:

A generic picture in $(k-1)$-space of an incidence structure $S$ lifts to a sharp scene in $k$-space if and only if $i^{\prime} \leq v^{\prime}+k f-(k+1)$ for all subsets $I^{\prime}$ on at least two faces.

In Section 5 we offer a second interpretation of these matroids as generalizations of Crapo's concurrence geometries [1]. For $k=2$, this matroid fits the task of realizing a configuration of lines and points with specified directions for the lines. The theorem then says that:

A configuration of lines is realizable with distinct vertices and general directions if and only if $i \leq a^{\prime}+2 b^{\prime}-3$ for all subsets of $i^{\prime}$ incidences on $a^{\prime}$ lines and $b^{\prime}$ points. 
Because such realizations with fixed directions represent parallel redrawings, this result has an additional interpretation as a general form of Laman's theorem [7]:

The counts $i=a+2 b-3$ and $i^{\prime} \leq a^{\prime}+2 b^{\prime}-3$ for all nonempty subsets $I^{\prime}$ characterize minimal infinitesimally rigid plane frameworks with lines of bars and joints at the intersections.

With this corollary we also settle a conjecture characterizing the rigid frameworks in the plane formed by long rods and pins (more than two per rod).

This paper has a number of connections, in its roots and in some specific results, with the pioneering work of Henry Crapo. The results of Section 2 turned out to be a reformulation of his work on general position concurrence geometries [1] or geometries of circuits [2]. Both bodies of work have grown from the rich soil of the geometric study of statics and mechanics for frameworks. Crapo selected these areas of parallel redrawing and concurrence from our earlier joint work on Maxwell's theory of plane stresses and projected polyhedra [3]. As the results here confirm, this focus has brought forward combinatorial structures of fundamental geometric importance.

Our initial investigation of matroids defined by counting properties was joint work with Neil White [14], and we have continued to benefit from the stimulation and insights of this long-term collaboration [13], [15].

\section{The Picture Matroid for Elementary Scenes}

In this section we present a proof of Sugihara's conjecture for the simple case of elementary scenes in which all faces have exactly four vertices. For this case, we switch from the incidence structure $S=(V, F ; I)$ to a hypergraph $H=(V ; E)$, with each face replaced by the edge of its four incident vertices. For a set of $f^{\prime}$ faces and all the incident vertices, we find that the count simplifies (see Section 3):

$$
i^{\prime} \leq v^{\prime}+3 f^{\prime}-4 \quad \text { if and only if } \quad e^{\prime} \leq v^{\prime}-4
$$

\subsection{Pictures of Elementary Scenes}

Definition 2.1. A 4-uniform hypergraph is a hypergraph $H=(V, E)$ with a finite set $V$ (the vertices) and a collection $E$ (the edges) consisting of four distinct elements of $V$.

We allow several copies of a single subset to appear as distinct edges of the hypergraph. To minimize the notational inconvenience this will only be implicit in most of our notation.

Definition 2.2. Given a 4-uniform hypergraph $H=(V, E)$, a picture of $H$ is an assignment $P: V \rightarrow R^{2}$ with $p_{j}=\left(x_{j}, y_{j}\right)$. 
A scene over the picture $P$ is an assignment $Z: V \rightarrow R$ such that for each edge $e_{i}$ of the hypergraph, the corresponding points $q_{j}=\left(x_{j}, y_{j}, z_{j}\right)$ are in a plane of 3-space.

For any edge with vertices $v_{1}, v_{2}, v_{3}, v_{4}$ the condition imposed on the scene can be written:

$$
\operatorname{det}\left[\begin{array}{cccc}
x_{1} & x_{2} & x_{3} & x_{4} \\
y_{1} & y_{2} & y_{3} & y_{4} \\
z_{1} & z_{2} & z_{3} & z_{4} \\
1 & 1 & 1 & 1
\end{array}\right]=0
$$

For a fixed picture this gives one linear constraint on the unknowns $z_{i}$. We write the system of homogeneous linear equations for all the edges as $A(H, P) \times Z=0$.

Before we compute the rank of this matrix, we need some more notation. We assume that the vertices and edges of $H$ are ordered and define sign $(i, j)$ to be $(-1)^{m}$ if $v_{j}$ is the $(m+1)$ st vertex in edge $e_{i}$. For any matrix $X$ with $v$ columns $X\left(e_{i} / v_{j}\right)$ will mean the matrix with columns for all vertices in $e_{i}$ except $v_{j}$.

Definition 2.3. Given a 4-uniform hypergraph $H(V, E)$ and a $3 \times v$ matrix with columns $p_{i}=\left(x_{i}, y_{i}, 1\right)$ the matrix $A(H, P)$ is defined by

$$
d_{i j}= \begin{cases}\operatorname{sign}(i, j) \operatorname{det}\left(P\left(e_{i} / v_{j}\right)\right) & \text { if } v_{j} \in e_{i}, \\ 0 & \text { otherwise }\end{cases}
$$

Proposition 2.1. For a picture $P$ over the hypergraph $H$ the kernel of the matrix $A(H, P)$ is the space of scenes over the picture and the rows of $A(H, P)$ represent the linear constraints on the scenes.

Any picture will have the trivial scenes, with all vertices coplanar. Unless the vertices in the picture are collinear, these trivial scenes form a three-dimensional subspace. A sharp scene has distinct planes for each pair of faces-which means that there must be at least a four-dimensional space of scenes and this kernel, restricted to the coordinates for the vertices of any pair of spaces, must also be at least four-dimensional.

A count of the variables, equations, and the desired solution spaces for the matrix $A(H, P)$ suggests the following condition (if the rows are independent):

a picture for a hypergraph $H$ has a sharp scene if $e^{\prime} \leq v^{\prime}-4$ for all subsets $E^{\prime}$ of at least two edges.

This condition is also a necessary condition for some pictures over the hypergraph $H$. Specifically, a picture $P$ is a general picture if it has the minimum space of scenes (solution space) for each hypergraph on the vertices. (For example, if the entries in $P$ are algebraically independent numbers over the rationals then $P$ will be general.) For a general picture, each set of incidence constraints or rows of $A(H, P)$ will have the maximal rank. 


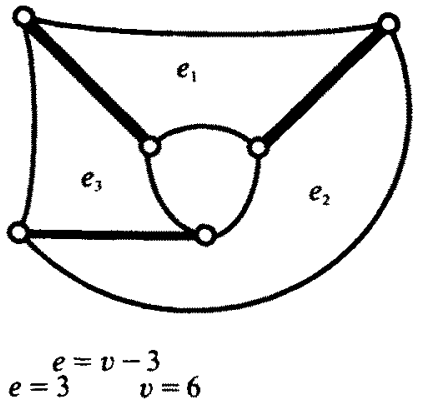

(a).

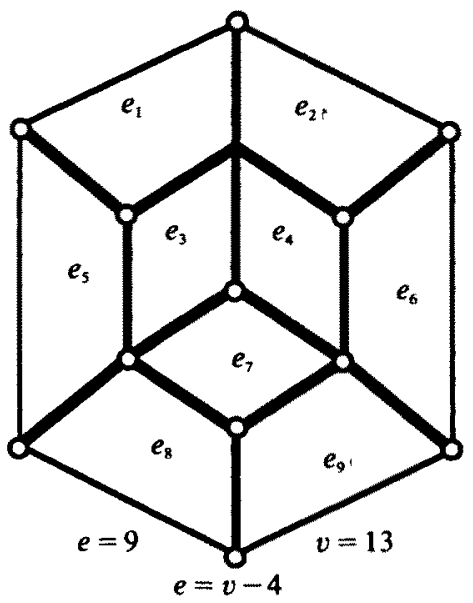

(b)

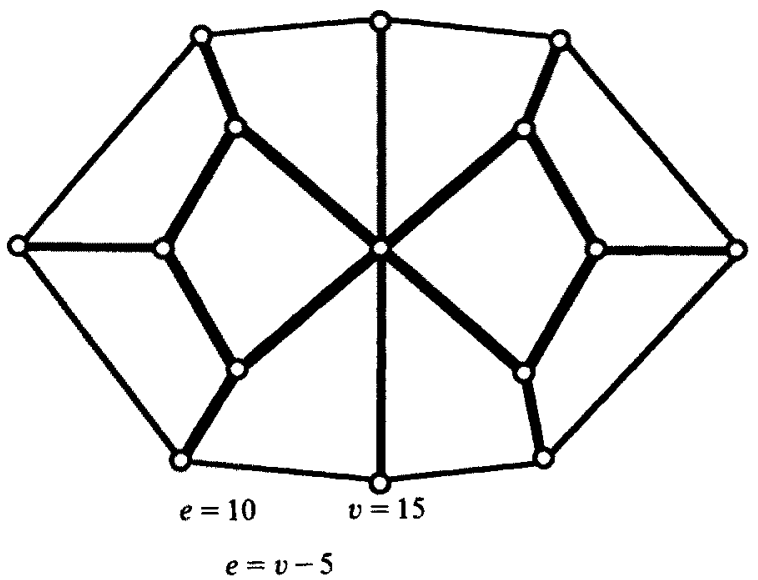

(c)

Fig. 1. The elementary scene shown in (a) is flat $(e=v-3)$, while the scenes shown in (b) and (c) are sharp ( $e^{\prime} \leq v^{\prime}-4$ for nonempty substructures).

Figure 1 shows some pictures of elementary scenes. The vertices are shown by circles, the edges by labeled polygons, with heavy lines separating two adjacent polygons. Figure 1(a) has no sharp scenes, while Fig. 1(b) has a four-dimensional space of scenes (almost all sharp), and Fig. 1(c) has a five-dimensional space of scenes.

Theorem 2.2.. A general picture for a 4-uniform hypergraph lifts to a sharp scene if and only if $e^{\prime} \leq v^{\prime}-4$ for all subsets of at least two edges.

This result is essentially a corollary to the following characterization of independent rows of $A(H, P)$. 
Theorem 2.3. For a general picture $P$ of a 4-uniform hypergraph $H$, the rows of $A(H, P)$ are independent if and only if $e^{\prime} \leq v^{\prime}-3$ for all subsets of at least one edge.

\subsection{Transversal Matroids}

Before we give the proofs, we need some basic counting properties for the rows of a related matrix which represents the transversal matroid (see Emonds [4] and Mirsky and Perfect [8] for more details on this matroid).

Definition 2.4. For a hypergraph $H$ and an $e \times v$ matrix $X$ of indeterminates $x_{i j}$ over the reals, the matrix $T(H, X)$ is the $e \times v$ matrix with entries

$$
t_{i j}= \begin{cases}x_{i j} & \text { if } v_{j} \in e_{i} \\ 0 & \text { otherwise }\end{cases}
$$

A variant on Hall's theorem [5] characterizes the independent sets of rows in $T(H, X)$.

Proposition 2.4. A set of edges $E$ is independent in the $T(H, X)$ if and only if for any subset $E^{\prime}$ of $E, e^{\prime} \leq v^{\prime}$.

Proof. For completeness we present a proof, due to Edmonds [4].

Assume that a set of rows is independent in $T(H, X)$. By counting the variables and equations, we clearly have $e^{\prime} \leq v^{\prime}$ for all subsets.

Assume that a set is dependent in $T(H, X)$. There must be a circuit $C$ of $k+1$ rows in the matrix $T(H, X)$. This in turn must contain a basis $C^{\prime}$ of $k$ independent columns. The row dependence gives a $(k+1)$-vector $Y$ such that $Y \times C^{\prime}=0$, with the vector composed of polynomials in the variables of $C^{\prime}$. Since $C^{\prime}$ is a column basis of $C, Y \times C=0$. If any column of $C$ not in $C^{\prime}$ contains nonzero entries, this would give a polynomial equation in these variables which has nonzero coefficients but is identically zero. We conclude that the remaining columns are zero, and that $e=k+1>k=v$.

Proposition 2.5. The matrix $T(H, X)$ has a solution space of exactly dimension $k$, which remains of dimension $k(k \neq 0)$, when restricted to any subset of $k$ vertices, if and only if, for any nonempty subset $E^{\prime}, e^{\prime} \leq v^{\prime}-k$.

Proof. If the hypergraph has $e^{\prime} \leq v^{\prime}-k$ then $E$ is independent and the matrix $T(H, X)$ has rank $v-k$. Thus there is a solution space of motions of dimension $k$. For any $k$ vertices, we can add singletons in these vertices to $H$, making $H^{*}=\left(V, E^{*}\right)$. This hypergraph satisfies $e^{*}=v$ and, for any subset $E^{\prime \prime}, e^{\prime \prime} \leq v^{\prime \prime}$. This independent set $E^{*}$ gives independent rows in the matrix $T\left(H^{*}, X^{*}\right)$ and blocks all of the motions of $T(H, X)$. Since these added rows were nonzero only on the chosen $k$ vertices, the solution space, restricted to these vertices, had dimension $k$. 
Conversely, assume that $T(H, X)$ has the solution space described. If any subset has $e^{*}>v^{*}-k$, then there is a set $V^{*}$ of at least $k$ vertices, containing a set $E^{\prime}$ of edges with $e^{\prime}=v^{\prime}-k+1$, and, for all proper subsets $E^{\prime \prime}, e^{\prime \prime} \leq v^{\prime \prime}-k$. With this hypergraph and its independent edges, the solution space has dimension $<k$. This contradicts the assumption.

\subsection{Proofs of the Theorems}

Proof of Theorem 2.3. (a) If $e^{\prime}>v^{\prime}-3$ for some subset, then the count of variables, equations, and solutions shows that the rows are dependent.

(b) Assume that the hypergraph satisfies the count $e^{\prime} \leq v^{\prime}-3$ for all nonempty subsets of edges-or any subset of at least three vertices. By Proposition 2.5, this hypergraph gives a matrix $T(H, Y)$ with a three-dimensional solution space, which remains of dimension 3 when restricted to any three vertices.

We use a general basis for a 3 -space of solutions as the rows of a $3 \times v$ matrix $M$ with columns $M_{j}$. Without loss of generality, we could assume that the third row of the matrix $M$ is $\left[\begin{array}{lll}1 & \cdots & 1\end{array}\right]$. This amounts to multiplying the $j$ th column of $T(H, Y)$ by $m_{3}$ and the column of $M$ by $1 / m_{3 j}$.

For any edge $f$, with vertices $v_{1}, \ldots, v_{4}$ (for simplicity), we have the three equations $\sum y_{i j} M_{j}=0$ (sum over the vertices of $f$ ). Since for any three vertices these columns are independent, and the $y_{y}$ are nonzero, up to multiplication of the row by a nonzero constant, we have

$$
y_{i j}=(-1)^{j-1} \operatorname{det}\left(M_{1} \cdots M_{j-1} \quad M_{j+1} \cdots M_{4}\right)=\operatorname{sign}(i, j) \operatorname{det}(M(f / v,)) .
$$

These values of $y_{i j}$ give the corresponding row of the matrix $A(H, M)$, a specialization of $A(H, X)$. Since these rows are independent in $T(H, Y)$, they remain independent in $A(H, M)$ and in $A(H, P)$ for any general picture.

Proof of Theorem 2.2. (a) Assume that the hypergraph satisfies the count. The solution set contains a nontrivial scene, by the count $e \leq v-4$.

Take any two edges $f=\left(v_{1}, v_{2}, v_{3}, v_{4}\right)$ and $g$. We want to prove that some scene over the picture gives distinct planes for these edges. By the count on the set $E^{\prime}=(f, g), g$ contains a distinct vertex-call it $v_{5}$. We add the edge $h=$ $\left(v_{1}, v_{2}, v_{3}, v_{5}\right)$ to $H$, creating a new hypergraph $H^{\prime}$. ( $h$ cannot be in $H$, since the doubleton $(f, h)$ does not have $e^{\prime \prime} \leq v^{\prime \prime}-4$.) This hypergraph now satisfies the hypothesis of Theorem 2.3. If any subset $E^{\prime \prime}$ in $H^{\prime}$ contains at least two edges of $H$, then the count, with at most one edge $h$ added gives $e^{\prime \prime} \leq v^{\prime \prime}-3$. If $E^{\prime \prime}$ contains only one edge of $H$, and $h$, then the count is also satisfied, since $h$ is not in $H$, and the doubleton has $e^{\prime \prime}=2=5-3=v^{\prime \prime}-3$. We conclude $h$ adds an independent row to $A(H, P)$-so it must remove a solution. This solution must place $v_{5}$ off the plane of $v_{1}, v_{2}, v_{3}$ as desired.

Since each pair of edges has distinct planes in some scene, an appropriate linear combination of these scenes will give distinct planes to the edges-and we have the desired sharp scene. 
(b) Assume that the hypergraph has sharp scenes for a general position picture. If any two edges lie in a subset $E^{\prime}$ with $e^{\prime} \geq v^{\prime}-3$, then there will be a subset $E^{*}$ of at least two edges, with $e^{*}=v^{*}-3$ and $e^{\prime \prime} \leq v^{\prime \prime}-3$ for all nonempty subsets. This subset generates independent rows of $A(H, P)$-and makes all these edges coplanar in all scenes. This is a contradiction.

\section{General Polyhedral Scenes}

A general scene has arbitrarily sized subsets of vertices which must remain coplanar. In addition, the objective is to characterize pictures which will have scenes with each such subset in a different plane. We recall the formal definitions, as found in [9] and [17].

Definition 3.1. An incidence structure is $S=(V, F ; I)$ where $V$ is the set of vertices, $F$ is a set of subsets of $V$, the faces, and $I$ is a set of ordered pairs in $V \times F$.

A picture $S(p)$ of the incidence structure $S$ is an assignment $p: V \rightarrow R^{2}$.

A scene $S(q, r)$ of the incidence structure $S$ is an assignment $q: V \rightarrow R^{3}$ and an assignment $r: F \rightarrow R^{3}$ such that for each $\left(v_{i}, f_{j}\right) \in I: r_{1} q_{1}+r_{2} q_{2}+q_{3}+r_{3}=0$.

A scene $S(q, r)$ lifts the picture $S(p)$ if the projection of $q$ down the last coordinates of all points gives $p$.

A scene is trivial if and only if for each pair of faces $f, f^{\prime}: r(f)=r\left(f^{\prime}\right)$.

A scene is sharp if and only if for each pair of faces $f, f^{\prime}: r(f) \neq r\left(f^{\prime}\right)$ and a picture is sharp if it lifts to a sharp scene.

The equation for an incidence in a scene says that the point $q_{i}$ is on the plane with coordinates $\left(r_{1}, r_{2}, 1, r_{3}\right)$. (With the entry 1 we have assumed that no plane is vertical.) For a fixed picture $p$ this is a linear equation in the unknowns $r_{1}, r_{2}, r_{3}, q_{3}$. These define a system of linear equations, and an $i$ by $(v+3 f)$ matrix $A(S, p)$. If $p$ is a set of algebraically independent numbers over the rationals, the independence of rows in $A(S, p)$ defines the picture matroid of $S$.

A simple count of the equations and the unknown leads to a natural conjecture:

A general position picture of an incidence structure $S=(V, F: I)$ is sharp if and only if $i^{\prime} \leq v^{\prime}+3 f^{\prime}+4$ for all substructures with at least two faces.

This is Sugihara's conjecture [9], for which he provided a proof covering some classes, such as subsets of a 3-connected spherical polyhedron.

Figure 2 shows some incidence structures of polyhedra which are covered by this criterion-so all general pictures will lift to sharp polyhedra. For spherical polyhedra the count $i \leq v+3 f-4$, and Euler's formula imply that the polyhedron must contain at least four triangles (Fig. 2(a)-(c)). A toroidal polyhedron meeting the count must contain at least eight triangles (Fig. 2(d)).

Definition 3.2. Given an incidence structure $S=(V, F ; I)$ and an ordering of the vertices, the derived 4-hypergraph $H(S)$ is the hypergraph $H(S)=\left(V, \bigcup E_{j}\right)$ 


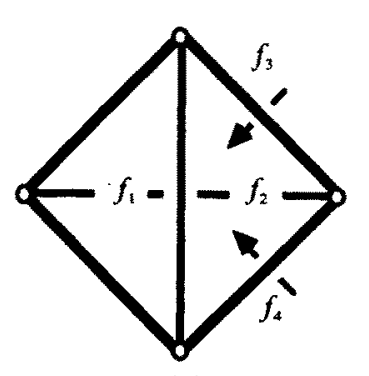

(a)

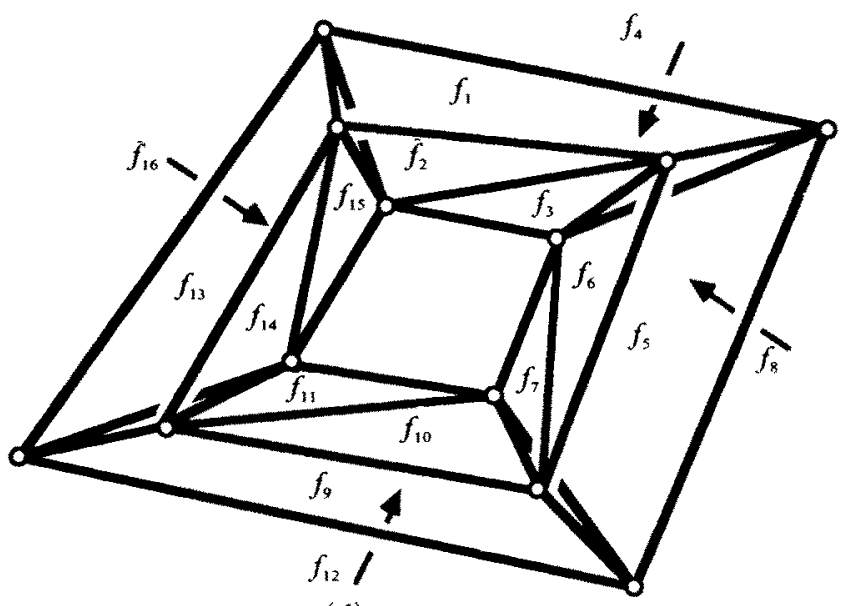

(d)

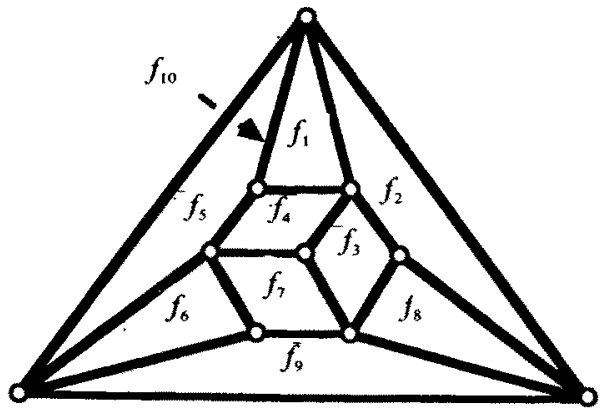

(b)

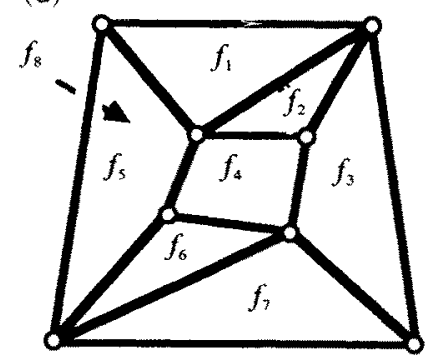

(c)

Fig. 2. By Sugihara's criteria, the general pictures of these spherical (a)-(c) and toroidal (d) polyhedra are sharp.

where for each face $f_{j}$ with vertices $v_{1}, v_{2}, v_{3}, \ldots, v_{3+m}$ the set $E_{j}$ consists of the edges $v_{1}, v_{2}, v_{3}, v_{3+n}$ for $1 \leq n \leq m$. If $f_{j}$ has less than four vertices then $E_{j}$ is empty. (If some 4-tuple appears in two distinct $E_{i}$, then we allow multiple copies of the edge. Such duplicate edges will be dependent in $A(H(S), p)$.)

Theorem 3.1. For an incidence structure $S$ the following are equivalent:

(a) The $S$ is independent in the picture matroid.

(b) For any nonempty subset $I^{\prime}$ of $I, i \leq v^{\prime}+3 f^{\prime}-3$.

(c) The derived 4-hypergraph $H(S)$ gives independent rows in $A(H(S), p)$ for general pictures $p$.

(d) The derived 4-hypergraph $H(S)$ satisfies $e^{\prime} \leq v^{\prime}-3$ for all nonempty subsets $E^{\prime}$ of edges.

Proof. The equivalence of $(c)$ and (d) is Theorem 2.3. We will show that (b) $\leftrightarrow$ (d) and that $(\mathrm{a}) \leftrightarrow(\mathrm{c})$.

We assume, for convenience, that all vertices are incident to some face. We also assume that each face is incident to at least three vertices, so that for general 
values of $p$, there will be at most one solution $r$ to make a scene with a given set $q$ of heights over these points. This can be accomplished by adding additional vertices which only lie on a single face. Such an addition will not change that character of the scene, and general points for these vertices can be added to any picture. The resulting additional rows in the matrix for a general picture of the incidence structure will therefore be independent of any other set of incidences, so property (a) will be unchanged.

These changes will not affect the definition of $H(S)$ (except to delete unattached vertices there). These changes will also not affect the count in (b), since we add one vertex and one incidence each time we fill in on a face.

$(\mathrm{d}) \rightarrow(\mathrm{b})$. For any incidence structure let $v\left(f_{i}\right)$ be the number of vertices incident with face $f_{j}$. For any $H(S)=\left(V, \bigcup E_{j}\right)$, writing $e_{j}$ for the size of $E_{k}$, we have

$$
i=\sum v\left(f_{j}\right)=\sum\left(e_{j}+3\right) \leq v+3 f-3 \quad \text { (sum over all faces). }
$$

Since this is true for all incidence structures defined by nonempty subsets of $I$, we have property (b).

(b) $\rightarrow(\mathrm{d})$. For each edge $e$ of $H(S)$ let the extension of $e$ be the entire set $E$, for some face $f_{j}$ containing the vertices. This may not be unique, but if some 4-tuple is actually contained in two faces, the incidence structure restricted to these vertices is dependent in the matrix and in the count. In such a case $H(S)$ was defined to have multiple edges-and it also violates the count.

We assume that (b) holds and that each edge lies in a unique $E_{j}$. If some set $E^{\prime}$ has $e^{\prime}>v^{\prime}-3$, and we extend each edge to get a set $E^{*}$ then $e^{*}>v^{*}-3$, since the extension adds at most one vertex for each added edge. For such an extended set $E^{*}$ :

$$
e^{*}=\sum\left(v\left(f_{j}\right)-3\right)=i-3 f^{*}>v^{*}-3
$$

From this contradiction we conclude that property (d) holds.

(a) $\leftrightarrow$ (c). Each scene $S(q, r)$ realizing a picture $p$ reduces to a unique scene over $H(S)(p)$. Conversely, each scene over $H(S)(p)$, with $p$ in general position (which will have no three points on a line) will extend to a unique set of values for $r$, since we assumed that each face contains at least three vertices and these points will determine a unique plane for the face. Therefore the two spaces of scenes have the same dimension $d$. Independence of $H(S)$ in $A(H(S), p)$ means $v-e=d$. Independence of $S$ in the picture matroid means $v+3 f-i=d$.

Corollary 3.2. For any incidence structure $S$, the following are equivalent:

(a) A general picture $S(p)$ is sharp.

(b) For any substructure $I^{\prime}$ with at least two faces, $i^{\prime} \leq v^{\prime}+3 f^{\prime}-4$.

Proof. (b) $\rightarrow$ (a). Assume that $S$ satisfies (b). By Theorem 3.1, the structure is independent and there is a nontrivial scene. If we take any two faces $f^{\prime}$ and $f^{\prime \prime}$ and add a new common vertex $w$, this creates a new structure $S^{*}$ with one more 
variable and two more equations. $S^{*}$ satisfies $i^{*} \leq v^{*}+3 f-3$, and this remains true for any proper subset. This new structure is also independent with a smaller dimensional space of scenes. If the two faces were already forced to share a common plane over $S(p)$, this would be impossible. We conclude that some scene places the faces into different planes. This is true for each pair of faces, so some appropriate linear combination of these scenes is the required sharp scene.

$(a) \rightarrow(b)$. Assume that (b) is false for $S$. Then there is a subset $I^{\prime}$ with more than one face and $i^{\prime}=v^{\prime}+2 f^{\prime \prime}-3$. Either $i^{\prime}=v^{\prime}+3 f-3$ and for all proper subsets $I^{\prime \prime}, i^{\prime \prime} \leq v^{\prime \prime}+3 f^{\prime \prime}-3$, or some nonempty subset has $i^{\prime \prime} \geq v^{\prime \prime}+3^{\prime \prime}-2$. This second possibility requires a circuit in the picture matroid. Such a circuit must have more than one face and removing one incidence gives an independent set of the same type. In either case this independent set will only allow a space of scenes of dimension 3 , so the faces must share a common plane in all scenes over a general point $p$. This makes (a) false as well.

Corollary 3.3. For any incidence structure $S$, and any $d \geq 1$ the following are equivalent:

(a) A general picture $S(p)$ has an affine space of scenes of dimension d, almost all sharp, with the first face fixed in the projection plane.

(b) $i=v+3 f-3-d$ and $i^{\prime} \leq v^{\prime}+3 f^{\prime}-4$ for any substructure $I^{\prime}$ with at least two faces.

Proof. Fixing the first plane in the projection plane removes a 3-space of solutions from the equations $A(H(S), P)$. The rest follows from Corollary 3.2.

\section{The $k$-Plane Matroid on Incidence Graphs}

Under all the language of three-dimensional scene analysis in Sections 2 and 3, we have a general result for any incidence graph $G=(A, B ; I)$ (usually thought of as the incidence graph of a hypergraph $H=(A ; B))$. We will restate the results for a general $k$.

Definition 4.1. For any incidence graph $G=(A, B ; I)$ (with $I \subset A \times B)$, the $k$-plane matroid at $B$ is the matroid defined on the incidences $I$ by:

a set $I^{\prime}$ is independent if and only if $i^{\prime \prime} \leq a^{\prime \prime}+k b^{\prime \prime}-k$ for all nonempty subsets $I^{\prime \prime}$.

As usual we order the vertices and the incidences in some arbitrary fashion.

Definition 4.2. Given an incidence graph $G=(A, B ; I)$ and $a(k-1) \times b$ matrix $X$ of distinct indeterminates, the matrix $B(G, X)$ is an $i \times(a+k b)$ matrix 
defined by

$$
\text { if } i_{j}=\left(a_{m}, b_{n}\right), \quad b_{j s}= \begin{cases}1 & \text { if } s=m, \\ x_{r m} & \text { if } r=s-(a+(n-1) k) \text { and } 1 \leq r \leq k-1, \\ 1 & \text { if } s=a+n k-1, \\ 0 & \text { otherwise. }\end{cases}
$$

If we let $Y=\left[\begin{array}{lllll}z_{1} \ldots z_{a} & y_{11} \ldots y_{1 k} & \ldots & y_{b 1} \ldots y_{b k}\end{array}\right]$ then the matrix equation $B(G, X) \times Y^{\prime}=0$ represents the system equations, one for each incidence $i_{j}=$ $\left(a_{m}, b_{n}\right)$,

$$
y_{n 1} x_{1 m}+\cdots+y_{n(k-1)} x_{(k-1) m}+z_{m}+y_{n k}=0 .
$$

For $k=3$ these are the same equations used to define scenes $Y$ over a picture $X$.

Definition 4.3 Given an incidence graph $G=(A, B ; I)$ and an ordering of the vertices, the derived $(k+1)$-hypergraph $H(G)$ is the hypergraph $H(G)=$ $\left(A, \cup E_{j}\right)$ where, for each vertex $b_{j}$ incident with vertices $a_{1}, \ldots, a_{k+m}$, the set $E_{j}$ consists of the edges $a_{1}, \ldots, a_{k}, a_{k+n}$ for $1 \leq n \leq m$. If $a_{1}$ has less than $k+1$ vertices then $E_{j}$ is empty. (If some edge appears in two distinct $E_{j}$, then we allow multiple copies of the edge. As we observed earlier such hypergraphs will be dependent in the extended depth $k$ matroid.)

Theorem 4.1. For any incidence graph $G=(A, B ; I)$, the rows of the matrix $B(G, E)$ are independent if and only if the corresponding incidences are independent in the $k$-plane matroid.

Proof. This is a simple translation of Theorem 3.1, with $k$ in place of 3 . All of the steps go through without change, reducing the problem through the depth $k$ matroid of the derived $(k+1)$-uniform hypergraph to the basic results on transversal methods.

For $k=0$ in this count, we have independence defined by $i^{\prime} \leq a^{\prime}$ for all nonempty subsets. This gives a trivial matroid, where incidences are independent if and only if they select different elements of $a$.

For $k=1$, we have independence defined by $i^{\prime} \leq a^{\prime}+b^{\prime}-1$, which returns us to the cycle or graphic matroid on the incidence graph, as a bipartite graph. Independent sets are forests and circuits are polygons in the graph.

For $k=2$, we have an important example in concurrence geometries which will be described in Section 5. This case can also be interpreted in terms of pictures on the line and scenes of lines and points in the plane.

For $k=3$, we have returned to the central example in scene analysis, as well as an interpretation in concurrence geometry.

For $k>3$, we have interpretations in terms of pictures in $(k-1)$-space and scenes in $k$-space [17, Section 7].

Definition 4.4. Given an incidence graph $G=(A, B ; I)$, a lifting $Y$ of the $k$-plane matroid is a solution of matrix equation $A(G, X) \times Y^{t}=0$. 
Theorem 4.2. For any incidence graph $G=(A, B ; I)$ and any $1 \leq m<k$, the following are equivalent:

(i) The space of liftings of the $k$-plane matroid of $H$ has dimension $m+k$, which remains of dimension $m+k$ when restricted to any subset of at least two vertices of $B$.

(ii) For all nonempty subsets $I^{\prime}$ of $I, i^{\prime} \leq a^{\prime}+k b^{\prime}-k-m$.

Proof. We proceed by induction on $m$.

For $m=1$, this is the content of Corollary 3.2 with 3 replaced by $k$. This requires no change in the proof.

The induction step from $m$ and $m+1$ is also a virtual copy of this proof. Adding any new vertex $a^{0}$ with edges to $b^{2}$ and $b^{3}$ will reduce the count, and the motion space to dimension $m+k$, by the count. This means that the edge removed an additional motion involving these two vertices from $b$. The rest now follows from the induction hypothesis.

Remark. For $m=k$, the count would prevent any edges and the entire statement would become trivial. For $m=0$, the space of lifting always have dimension at least $k$, so no condition exists on the incidence structure.

\section{Parallel Redrawings and Rigid Frameworks in the Plane}

\subsection{Concurrence Geometries and Parallel Redrawing}

In Section 2 the matrix $A(H, P)$ was developed for plane pictures of elementary scenes. The entries $\left(x_{i}, y_{1}, 1\right)$ in the matrix represented affine coordinates of points in 2-space or, equivalently, as projective coordinates of finite points in projective 2 -space. The solutions were interpreted as heights which placed the points into 3-space, with points of an edge sharing a plane.

In projective space such coordinates have a dual or polar interpretation. The original triples $\left(x_{i}, y_{i}, 1\right)$ also represent lines in a plane (not through the origin). The "lifting" extends this configuration to a set of planes in 3-space with the given intersections on a fixed cutting plane $z=0$. The equation for an edge constrains the four planes of the edge to share a point in 3-space. This interpretation is an example of Crapo's concurrence geometries [1]. It was in this geometric setting that the matrix $A(H, X)$ first appeared and a version of Theorem 2.3 was proven. The incidence structures of Section 3 and 4 also have a consistent interpretation as concurrent hyperplanes in a space, so the $k$-plane matroid generalizes the concurrence geometries.

If the sectioning plane (dual to the picture) is placed projectively as the plane at infinity, then the "picture" records the directions of the planes and the "liftings" form a space of parallel redrawings of the configuration of planes and points. For the faces of a polyhedron, such parallel redrawings have a number of applications to the study of Minkowski sums, infinitesimal rigidity, and the design of patterns of dihedral angles in 3-space [18] and in higher dimensions. 
For the case $k=2$, the analogous interpretation fits parallel redrawings of a set of lines in the plane with prescribed incidences, and directions. This subject has a long history dating back to the last century. We offer a brief statement and application of our results for this special case.

Definition 5.1. A plane configuration is an incidence graph $G=(A, B ; I)$ with an assignment of a normal direction $\left(f_{i}, g_{i}\right)$ to each line $a_{i}$.

A parallel redrawing is an assignment of a triple $\left(f_{i}, g_{i}, h_{i}\right)$ to each line $a_{i}$ a plane position $\left(x_{j}, y_{j}\right)$ to each point $b_{j}$, such that, for each edge $\left(a_{i}, b_{j}\right)$ in $I$,

$$
f_{i} x_{j}+g_{i} y_{j}+h_{i}=0 \text {. }
$$

A parallel redrawing is trivial if all points have the same position. A parallel redrawing is proper if distinct points have separate positions.

Theorem 5.1. Almost all plane configurations on an incidence graph $G=(A, B ; I)$ will have proper parallel redrawings if and only if $i^{\prime} \leq a^{\prime}+2 b^{\prime}-3$ for all nonempty subsets $I^{\prime}$.

Proof. The parallel redrawings of the configuration are the solutions of the matrix $A(G, P)$. The result is a simple restatement of Theorem 4.1.

Figure 3(a) and (b) shows general configurations with proper parallel redrawings $\left(i^{\prime} \leq a^{\prime}+2 b^{\prime}-3\right)$. The configuration in Fig. $3(c)$ is special, since general configurations on this graph will have only trivial parallel redrawings $(i=a+2 b-2)$.

\subsection{Bar and Joint Frameworks}

This result has important corollaries in the theory of plane bar and joint frameworks.

Definition 5.2. A bar and joint framework in the plane is a graph $G=(V, E)$ with as assignment $P$ of a point $p_{i} \in R^{2}$ to each vertex $v_{i}$ such that $p_{i} \neq p_{j}$ if $(i, j) \in E$.

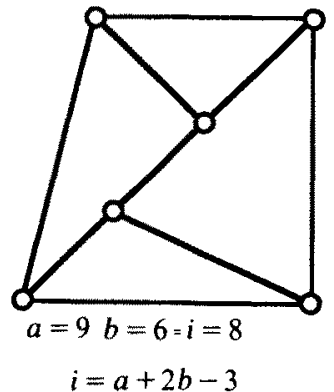

(a)

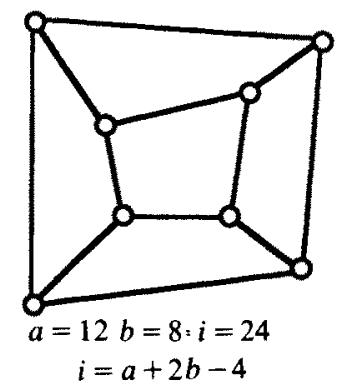

(b)

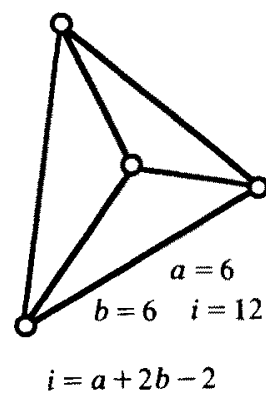

(c)

Fig. 3. By their counts, the configurations shown in (a) and (b) are general, while the configuration in (c) must be special, since they all have nontrivial parallel redrawings-the dilations. 
An infinitesimal motion of a bar and joint framework $(G, P)$ is an assignment $M$ of a vector $m_{i} \in R^{2}$ to each vertex $v_{i}$ such that, for each edge $(i, j)$, we have

$$
\left(p_{i}-p_{j}\right) \cdot\left(m_{\mathrm{r}}-m_{j}\right)=0 \text {. }
$$

A bar and joint framework in the plane, with more than one joint, is infinitesimally rigid if the space of infinitesimal motions has dimension 3 (the Euclidean motions).

A bar and joint framework in the plane defines a configuration of lines for the bars and points for the joints. Because we insist that vertices be distinct, the framework itself will be a proper parallel redrawing. By an old engineering technique, each parallel redrawing of a framework corresponds to an infinitesimal motion of the framework corresponds to an infinitesimal motion of the framework [2], [3], [16]. Each parallel redrawing gives a new position $p_{j}^{\prime}$ for the joint $p_{j}$, and the corresponding velocities are the vectors $p_{j}^{\prime}-p_{j}$ turned $90^{\circ}$ clockwise (Fig. $4(a)$ and (b)). In particular, the trivial parallel redrawings obtained by translation and dilation of any proper drawing correspond to the trivial motions of translation and rotation of the framework. (Note that for a trivial drawing there will be no

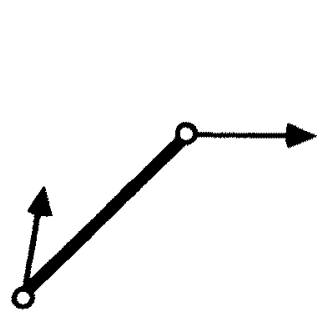

(a)

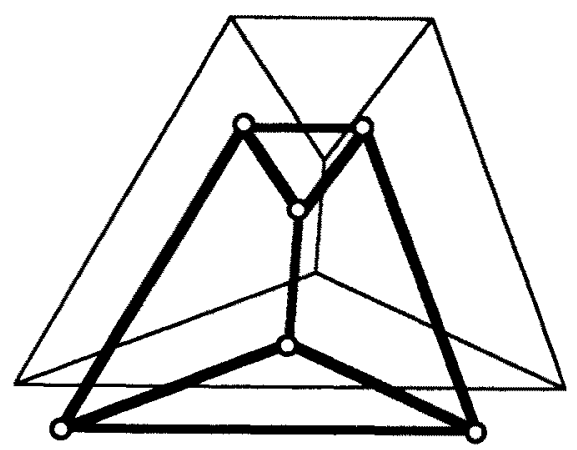

(c)

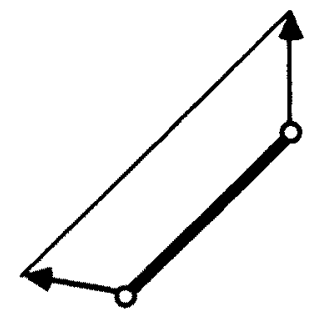

(b)

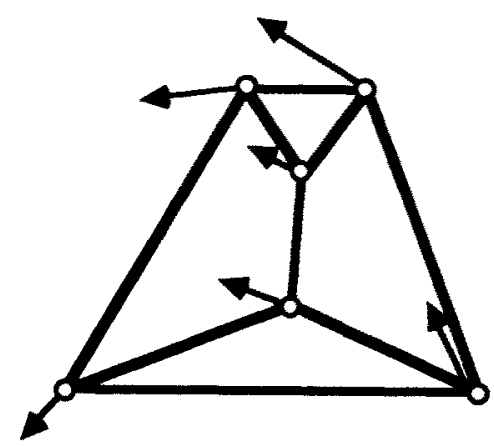

(d)

Fig. 4. An infinitesimal motion of a framework (a), (d) corresponds to a parallel redrawing of the configuration (b), (c). 
dilations.) Nontrivial redrawings of the framework (Fig. 4(c)) will correspond to nontrivial infinitesimal motion (Fig. 4(d)).

A framework with a subset of bars forming a collinear tree for each line will form a general configuration of lines and points, with each collinear tree as a single "face".

Definition 5.3. A bar and joint framework realizes an incidence graph $G=$ $(A, B ; I)$ if there is a joint for each vertex in $B$, and each vertex in $A$ is replaced by a connected chain (a tree) of collinear bars among all vertices incident with the line.

Theorem 5.2. An incidence graph $G=(A, B ; I)$ has a realization as an isostatic (minimal infinitesimally rigid) bar and joint framework in the plane if and only if $i=a+2 b-3$, and for any proper subset $I^{\prime}: i^{\prime} \leq a^{\prime}+2 b^{\prime}-3$.

Proof. If the graph has the required count, then any general position configuration will have a proper parallel redrawing. This can be used to construct the framework. Since this redrawing has a space of parallel redrawings of exactly dimension 3 , by Theorems 5.1 and 4.2 , the corresponding framework has a space of infinitesimal motions of exactly dimension 3 . This means that the framework is infinitesimally rigid.

If there is a minimal infinitesimally rigid framework realizing this graph, then the set $U$ of bars gives $2 v-3$ independent equations on the $2 v$ unknown velocities of the vertices in the plane. If a line $a_{i}$ contains val $\left(a_{i}\right)$ points then the framework has val $\left(a_{i}\right)-1$ bars along this line. If we take a set $I$ of edges in the incidence graph, we find that $u=2 v-m$ if and only if $i=a+2 b-m$ for any $m$. Since independence of the bar equations requires $u \leq 2 v-3$, we conclude that the incidence graph has the required count.

Remark. If each line has only two incident vertices, we have a general framework and this is Laman's theorem [7]. We give a related, but simpler proof of this theorem in [16]. In general, our stronger result guarantees that certain sets of edges can be in forced collinear without loosing the desired rank of the rigidity matrix which records the bar equations. In this plane setting there are a wealth of examples and further connections with fields such as plane statics which we cannot begin to describe here. Some examples are shown in Fig. 5 . We note that Fig. 5(c) shows a nonrigid realization (by the substructure shown), although a general framework on the underlying $K_{3,3}$ is infinitesimally rigid.

A rereading of the proof gives the following corollary, which we shall need shortly.

Corollary 5.3. An incidence graph $G=(A, B ; I)$ has a realization as an independent bar and joint framework in the plane if and only if, for any nonempty subset $I^{\prime}, i^{\prime} \leq a^{\prime}+2 b^{\prime}-3$. 


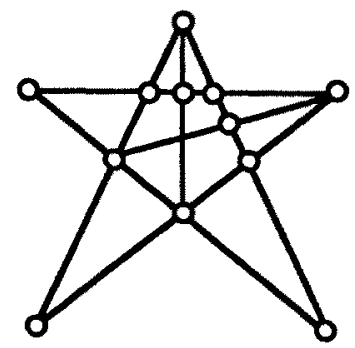

$a=7 \quad b=12 \quad i=28$

$$
i=a+2 b-3
$$

(a)

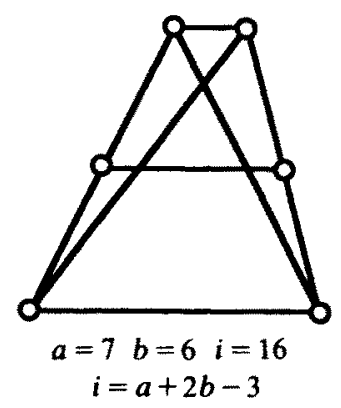

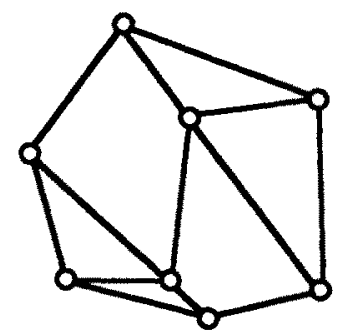

$a=11 \quad b=8 \quad i=24$

$i=a+2 b-3$

(b)

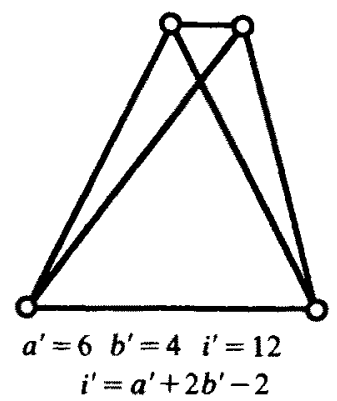

(c)

Fig. 5. Some examples of plane frameworks with collinear bars are generally infinitesimally rigid $(a, b)$, while others always have nontrivial infinitesimal motions $(c)$.

\subsection{Bar and Body Frameworks in the Plane}

This result also settles a conjecture about body and joint frameworks in the plane [11, Conjecture 2]. We need one more definition.

Definition 5.4. An identified body and joint framework in the plane is an incidence graph $G=(B, J ; I)$ with an assignment $P$ of a point $p_{i} \in R^{2}$ to each vertex $j_{i}$ in $J$.

An identified body and joint framework is infinitesimally rigid if there is a replacement of each body $b_{k}$ in $B$ by an isostatic plane bar and joint framework which includes the joint $p_{i}$ if $\left(b_{k}, j_{i}\right) \in I$ (and includes at least two joints in total), and this total replacement bar and joint framework is infinitesimally rigid.

An identified body and joint framework is independent if there is a replacement framework, with isostatic frameworks for the bodies, which is independent.

Remark. This definition of infinitesimal rigidity is just a short cut to avoid the usual descriptions of centers of motion and projective transformations to velocities [11], [15]. It is equivalent to the usual definition, but it is better suited to the next proof. 
Theorem 5.4. Given an incidence graph $G=(B, J ; I)$ the following are equivalent:

(i) $G$ has a realization as an independent (isostatic) identified body and joint framework in the plane.

(ii) $G$ satisfies $2 i \leq 3 b+2 j-3(=)$ and, for every subset of bodies and induced subgraph of attached joints, $2 i^{\prime} \leq 3 b^{\prime}+2 j^{\prime}-3$.

(iii) $G$ has an independent (isostatic) realization as an identified body and joint framework in the plane such that each body has all its joints collinear.

Proof. (iii) $\rightarrow$ (i) is trivial.

(i) $\rightarrow$ (ii). This is a consequence of Laman's theorem, using a simple counting argument [11]. If any body has less than two joints, we simply add new joints incident only to this body. This will not affect the count or the independence. Each body $b_{k}$ is replaced by an isostatic framework on the $j_{k}$ vertices attached to the body, and an appropriate graph with $2 j_{k}-3$ edges. This creates a total graph $G^{*}$ with a count

$$
\sum e_{k}=e^{*} \leq 2 v^{*}-3 \leq 2 j-3 \text {. }
$$

We also know that

$$
2 i=2\left(\sum j_{k}\right)=\sum\left(e_{k}+3\right)=\sum e_{k}+3 b \leqq 3 b+2 j-3 .
$$

Since every subset is also independent, the similar count holds for all nonempty subsets of bodies. If the framework was isostatic, then clearly equality holds for the graph.

(ii) $\rightarrow$ (iii). Each body in the graph with $j_{k}$ attached vertices will be replaced by a line of $j_{k}$ joints and an extra joint off this line, attached to each of these $j_{k}$ joints by a new line (Fig. 6). This creates a new incidence graph $G^{0}=\left(A^{0}, B^{0} ; I^{0}\right)$ of lines $A$ and joints $B$, to which we can apply Theorem 5.2. For any subgraph of $G$ we assume that $2 i^{\prime}=3 b^{\prime}+2 j^{\prime}-3$. In the new graph $G^{0}$ each incidence in $I$ places a joint on two lines, while each new new joint for a body is on $j_{k}$ lines, so

$$
e=2 i+\sum j_{k} \leq 3 b+2 j-3+\sum j_{k}=\left(\sum j_{k}+b\right)+2(b+j)-3=a^{0}+2 b^{0}-3 .
$$

Since the same count holds for all subsets of $b^{\prime}$ bodies, it holds for any subgraph of $G^{0}$ in which a body with some lines has all the lines present.

Assume some other substructure, containing only some of the lines and vertices for a body, fails the condition $e^{\prime} \leq a^{\prime}+2 b^{\prime}-3$. If a line does not have at least two joints, then it can be dropped, maintaining this failure. If this substructure contains at least one line (and two joints) from a body, then we can fill out this body by adding:

(a) The special vertex for the body, if missing, with its lines to all present vertices (one vertex, $n$ lines, and $2 n$ incidences, $n=2$ ).

(b) The basic line of the body, if missing (one line and at least one incidence).

(c) New vertices along the basic line of the body, and the line to the body vertex (one vertex, one line, and three incidences). 


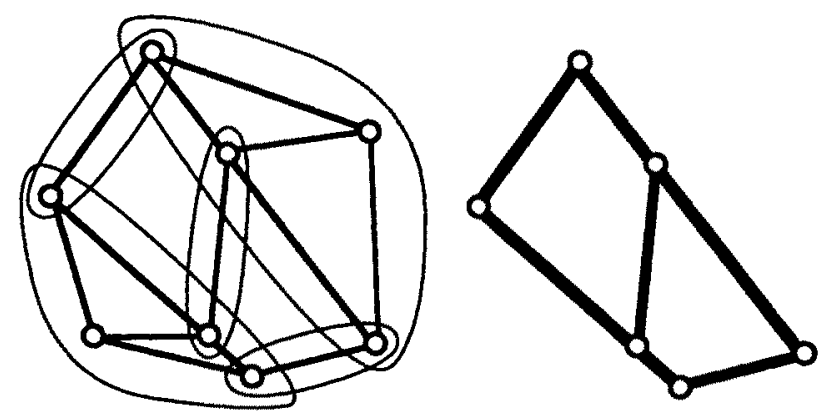

(a)

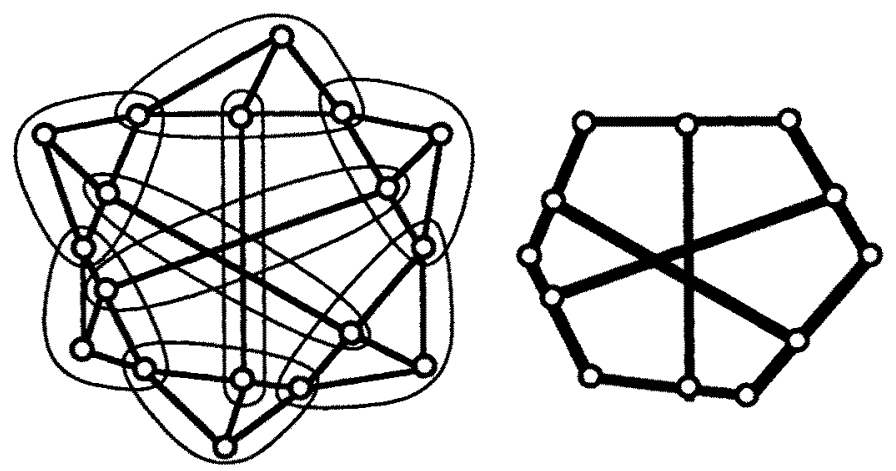

(b)

Fig. 6. Bodies with all points collinear (heavy lines) are equivalent to frameworks with an added joint for each body.

(d) Missing lines between joints on the basic line and the special vertex for the body (one line and two incidences).

These additions can only maintain or increase any failure of the count. Since full subsets satisfy the count, we conclude that all nonempty subsets do also.

By Corollary 5.3, the graph $G^{0}$ has a realization as an independent bar and joint framework. This is the required realization of $G$ with all the original joints for any body collinear.

Remark. This theorem is the plane analogue of an important conjecture about spatial structures. The appropriate definitions are given in [11].

Conjecture. A bipartite graph can be realized in 3-space as an infinitesimally rigid identified body and hinge structure if and only if it can be realized as an infinitesimally rigid identified body and hinge structure with all hinges of each body in a single plane. 
For the special case where each hinge connects only two bodies, we do have a characterization of the graphs [11], but the methods used here do not extend to solve even this special case of the conjecture.

We pointed out that, for $k=3$, Theorem 4.1 describes spatial configurations of planes and points which have parallel redrawings. However all motions of spatial bar and joint frameworks cannot be expressed as parallel redrawings. In fact, the spatial analogue of Laman's theorem is a central unsolved problem which cannot be approached by the path used here [12]. However, the problem of parallel redrawings of line configurations in $k$-space can be studied by these matroids. The resulting theorems apply both to infinitesimal motions of frameworks in $k$-space and to Minkowski sums of polytopes in $k$-space [18].

\subsection{Special Position Problems}

We have concentrated on the structures of general values of the variables. For some special values (the special positions) the rank of some subsets will change. The given matrices still represent the underlying engineering problem, but the independence or dependence of a set of rows will depend on the geometry of the points, as well as the count of the original structure.

From the pattern of representation given in Sections $2-4$, the set of special positions for the vertices of any hypergraph is projectively invariant. Thus the geometry of these specializations is projective geometry. Crapo has begun a study of this geometry (for the elementary scenes and their analogues) under the title of the circuit geometry of the configuration of points in projective $(k-1)$-space [2].

For both scene analysis in space and parallel redrawing in the plane there is an extensive literature on special positions. An enormous number of pretty geometric results and exciting unsolved problems lie in this direction. The pattern of our representing matrix, and the polynomials obtained from determinants of its minors can be used to develop an algebraic geometry of the special positions which make sets of these determinants $=0$. A model for this type of study is provided by [13] and [15] where special positions of frameworks are analyzed.

\section{References}

1. H. Crapo, Concurrence geometries, Adv. in Math. $\$ 4$ (1984), 278-301.

2. H. Crapo, The combinatorial theory of structures, in Matroid Theory (L. Lovasz and A. Recski, eds.), 107-213, Colloquia Mathematica Societatis János Bolyai, Vol. 40, North-Holland, New York, 1985.

3. H. Crapo and W. Whiteley, Plane stresses and projected polyhedra, Preprint, Champlain Regional College, 900 Riverside Drive, St. Lambert, Quebec, J4P-3P2, 1986.

4. J. Edmonds, Systems of distinct representatives and linear algebra, J. Res. Nat. Bur. Standards B 71 (1967), 241-245.

5. P. Hall, On representatives of subsets, $J$. London Math. Soc. 10 (1935), 26-30.

6. H. Imai, On combinatorial structure of line drawings of polyhedra, Discrete Appl. Math. 10 (1985), 79-92. 
7. G. Laman, On graphs and rigidity of plane skeletal structures, J. Engrg. Math. 4 (1970), 331-340.

8. L. Mirsky and H. Perfect, Applications of the notion of independence to problems of combinatorial analysis, J. Combin. Theory Ser, B 2 (1967), 327-335.

9. K. Sugihara, An algebraic and combinatorial approach to the analysis of line drawings of polyhedra, Discrete Appl. Math. 9 (1984), 77-104.

10. K. Sugihara, Machine Interpretation of Line Drawings, Artificial Intelligence Series, MIT Press, Cambridge, MA, 1986.

11. T.-S. Tay and W. Whiteley, Recent progress in the generic rigidity of structures, Structural Topology 9 (1984), 31-38.

12. T.S. Tay and W. Whiteley, Generating all isostatic frameworks, Structural Topology 11 (1985), 21-68.

13. N. White and $W$. Whiteley, The algebraic geometry of stresses in frameworks, SIAM J. Algebraic Discrete Methods 4 (1983), 481-511.

14. N. White and $W$. Whiteley, $A$ class of matroids defined on graphs and hypergraphs by counting properties, Preprint, Department of Mathematics, University of Florida, Gainesville, FL 32611, 1984.

15. N. White and $W$. Whiteley, The algebraic geometry of motions in bar and body frameworks, SIAM J. Algebraic Discrete Methods 8 (1987), 1-32.

16. W. Whiteley, The union of matroids and the rigidity of frameworks, Preprint, Champlain Regional College, 900 Riverside Drive, St. Lambert, Quebec, J4P-3P2, 1983.

17. W. Whiteley, A correspondence between scene analysis and motions of frameworks, Discrete Appl. Math. 9 (1984), 269-295.

18. W. Whiteley, Parallel redrawings of configurations in 3-space, Preprint, Champlain Regional College, 900 Riverside Drive, St. Lambert, Quebec J4P-3P2, 1986.

Received April 30, 1986, and in revised form February 9, 1987. 